\title{
Fully integrated optical polarimeter ${ }^{1}$
}

\author{
Ton Koster and Paul Lambeck
}

Lightwave Devices Group (LDG), Mesa+ Research Institute, PO Box 217, 7500 AE Enschede, The Netherlands, t.m.koster@el.utwente.nl

Abstract- The principle, the design and the experimental results of a fully integrated optical polarimeter are presented.

\section{Introduction}

Although equipped with an excellent sensory system, a human being is for many sensor applications not the preferred interrogation system. For these applications, micro systems technology, amongst them integrated optics, offers a range of solutions. Over the years, these integrated optical systems have proven to be sensitive, reliable and compact sensors for the determination of many types of measurands.

Generally, in these integrated optical sensors the measurand affects some modal characteristic of a guided mode in a waveguide. Then, by incorporation of the sensing region into an appropriate optical circuit, these changes in the modal characteristic are converted into intensity changes which can be measured in the electrical domain by virtue of a detector.

The fully integrated optical polarimeter presented in this contribution is such a sensing circuit. The here described device is the first polarimeter in which all purely optical functionalities have been integrated in a single optical chip, resulting in a more compact and stable system compared to the hybrid structures reported on up to now [1][2][3][4][5][6]. In the following sections, first the principle of the polarimeter, next the design and finally experimental data on the performance of the polarimeter and the individual optical functionalities are presented.

2. Principle of the Polarimeter

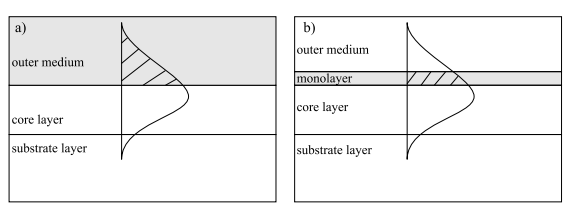

Figure 1. Two types of evanescent field sensing a) Homogeneous, where all of the evanescent field is confined in the sensing layer, and b) surface evanescent field sensing, where the evanescent field penetrates significantly in the environment on top of the sensing layer.

The sensing principle used is evanescent field sensing, as shown in figure 1. Here, by interaction with the evanescent field the measurand ' $m$ ' affects the properties of a guided mode in a waveguide directly or indirectly, in the latter case, e.g. using chemo-optical transduction layers in which (selective) receptor molecules have been incorporated. If it is the phase $\phi$ of the mode that is affected, the sensor is called a refractometer. Several circuits can be used to convert a change $\Delta \phi(m)$ in the phase into a change in the optical power $P_{\text {opt }}$, such as interferometric ones of which the Mach Zehnder Interferometer (MZI [7]) is the best known. In an MZI, the difference in the phase between a mode in the sensing branch and a mode propagating through the reference branch, $\Delta \phi_{s}(m)-\Delta \phi_{r}$, results in a sinusoidal relationship between $P_{\text {opt }}$ and $\Delta \phi_{s}(m)$, see figure 2 . In a

${ }^{1}$ This research was funded by STW, the Dutch technology foundation TEL 44.3474 


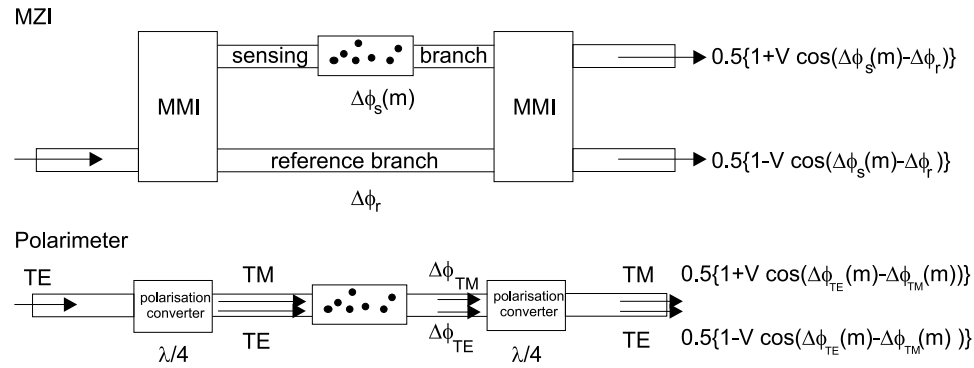

Figure 2. Functional layout of an MZI and a polarimeter, showing the similarities in functionalities and output signals between both sensing circuits. $\mathrm{V}$ is the fringe visibility.

polarimeter, a TE and TM mode propagating through the same waveguide both experience a (different) phase shift, $\Delta \phi_{T E}(m)$ and $\Delta \phi_{T M}(m)$. After the sensing function, using an integrated optical $\lambda / 4$ wave plate, a TE and TM polarised output signal are obtained of which the intensity is a function of the difference $\Delta \phi_{T E}-\Delta \phi_{T M}$.

\section{Physical Design of the polarimeter}

The circuit will be fabricated in the SiON technology as developed in our laboratory [8], which permits the realisation of highly sensitive and cheap sensors. The topview of the device and the waveguide crosssections at relevant positions are shown in figure 3. From the left to the right, the device consists of a passive polarisation converter [9], which couples half of the launched $T E_{00}$ mode power to the $T M_{00}$ mode. Next, both modes propagate through the sensing function, where they experience the measurand induced phase shift, after which the modes interfere in a

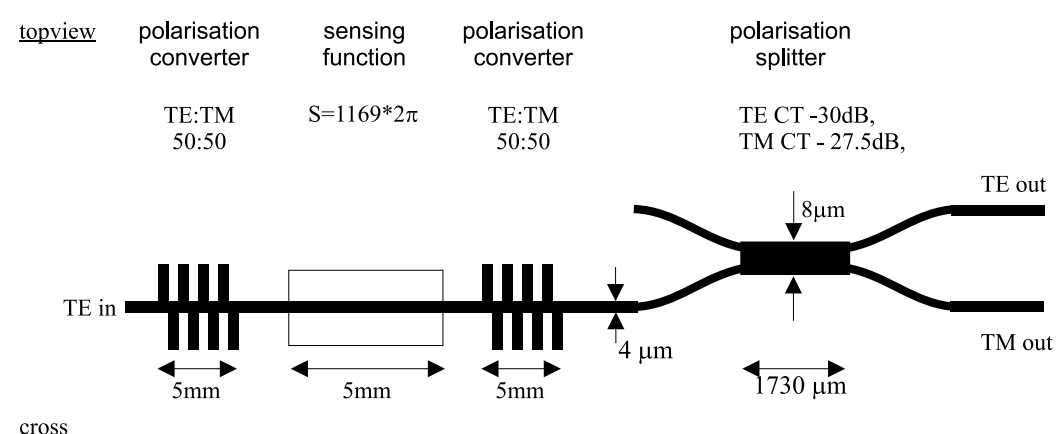

$\underline{\text { sections }}$
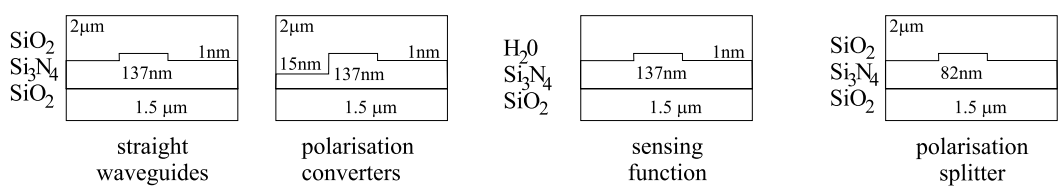

Figure 3. Topview and waveguide crosssections of the polarimeter. Also, the expected (calculated) performance of the individual functionalities are given. The total device area is approximately $0.1 * 25$ square $\mathrm{mm}$. 
second polarisation converter. The output TE and TM mode of this converter are separated into different output waveguides using a two mode interferometer polarisation splitter, resulting (for a TE polarised input signal with an optical power $\left.P_{i n}\right)$ in the output signals

$$
\begin{aligned}
P_{T E} & =\frac{1}{2} P_{i n}\left\{1-V \cos \left\{\Delta \phi_{T E}(m)-\Delta \phi_{T M}(m)\right\}\right\} \\
P_{T M} & =\frac{1}{2} P_{i n}\left\{1+V \cos \left\{\Delta \phi_{T E}(m)-\Delta \phi_{T M}(m)\right\}\right\}
\end{aligned}
$$

where $\mathrm{V}$ is the fringe visibility. Although in principle only one of the modes has to be detected in order to determine the measurand, for future implementation of signal processing techniques which reduce the influence of laser noise and increase the resolution of the sensor, we focussed on detecting both modes. A more detailed description and analysis of the individual functionalities and the complete sensor can be found in [10].

\section{Device FABrication}

The device is fabricated on a $\mathrm{Si}$ wafer using a $\mathrm{SiO}_{2}-\mathrm{Si}_{3} \mathrm{~N}_{4}-\mathrm{SiO}_{2}$ layer sandwich. Layers are growth thermally $\left(\mathrm{SiO}_{2}\right.$ substrate), by low pressure chemical vapour deposition $\left(\mathrm{Si}_{3} \mathrm{~N}_{4}\right.$ core $)$ and by plasma enhanced chemical vapour deposition $\left(\mathrm{SiO}_{2}\right.$ protective layer). The waveguide circuit, the required tapering of the core layer and a sensing window in the protective $\mathrm{SiO}_{2}$ layer are realised using BHF etching. The grating structure of the polarisation converter is realised using a double masking technique [9] and RIE.

\section{EXPERIMENTAL RESULTS}

Besides the complete polarimeter, the individual subfunctions have also been fabricated separately in order to characterise their individual performance. The results of this characterisation are shown in the table. In the second column expected performance characteristics (if calculated) are given, in the third column the experimentally determined values.

\begin{tabular}{|l|l|l|}
\hline & calculated & experimental \\
\hline $\begin{array}{l}\text { Channel waveguides } \\
\text { propagation losses }\end{array}$ & $1.6 \pm 0.1 \mathrm{~dB} / \mathrm{cm}(\mathrm{TE}$ and $\mathrm{TM})$ \\
\hline $\begin{array}{l}\text { Sensing function } \\
\text { phase sensitivity }\end{array}$ & $1169 * 2 \pi$ & $1096 * 2 \pi$ \\
\hline $\begin{array}{l}\text { Polarisation converter } \\
\text { average resonance wavelength } \\
\text { functional losses }\end{array}$ & $655 \pm 3.7 \mathrm{~nm}$ & $655.3 \pm 4.3 \mathrm{~nm}$ \\
\hline $\begin{array}{l}\text { Polarisation splitter } \\
\text { TE crosstalk }\end{array}$ & $-30 \mathrm{~dB}(\mathrm{~L}=1.73 \mathrm{~mm})$ & $-27 \mathrm{~dB}(\mathrm{~L}=1.69 \mathrm{~mm})$ \\
TM crosstalk & $-27 \mathrm{~dB}(\mathrm{~L}=1.73 \mathrm{~mm})$ & $-24 \mathrm{~dB}(\mathrm{~L}=1.69 \mathrm{~mm})$ \\
\hline
\end{tabular}

For the complete sensor, a fringe visibility of 0.8 for the TE and TM output signal has been determined by tuning the operating wavelength to the resonance wavelength of the fabricated converters. As a practical application, where the sensory system of many humans shows a lack of sensitivity which is sometimes misused by bartenders, consider the case of discriminating between tap water and mineral water. In an experiment, while measuring the TE output signal, using a flow injection system the polarimeter has been exposed to alternating flows of tap and mineral water. In figure 4 , it can be seen that the polarimeter can clearly discriminate between both types of water. The difference in the output signal corresponds to a refractive index difference of $6.8 * 10^{-5}$. 


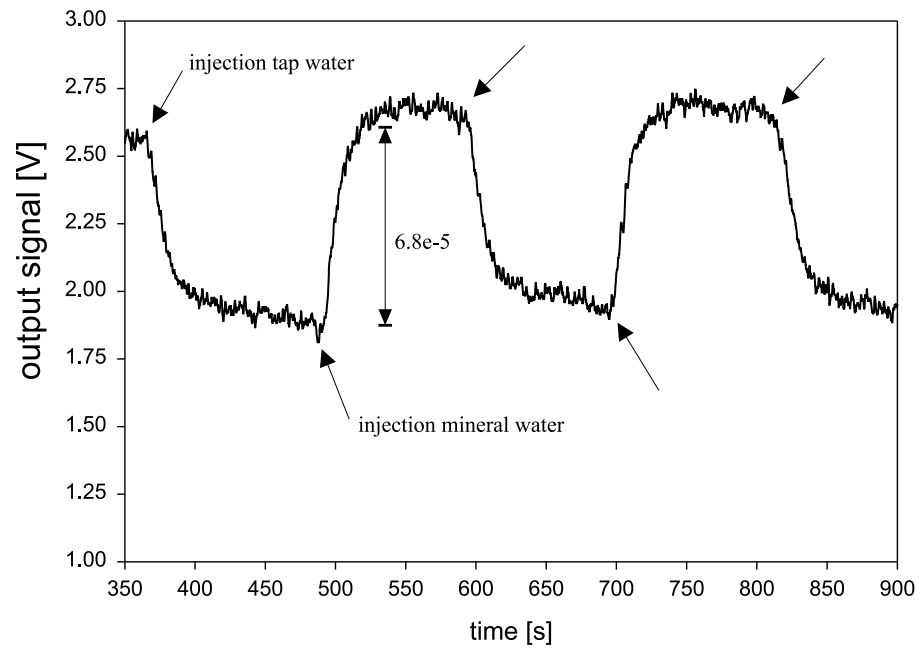

Figure 4. The output signal as a function of the type of water (tap or mineral). The arrows indicate the switching between a flow of mineral to a flow of tap water or vice versa.

\section{Summary}

The principle, the design and experimental results of a fully integrated optical polarimeter have been presented. All optical functionalities, a sensing function, two polarisation converters and a polarisation splitter are integrated in a single optical circuit. Currently, further integration of the device with fibre to chip couplers and monolithically integrated photodiodes is investigated.

\section{REFERENCES}

[1] C. Stamm and W. Lukosz. Integrated optical difference interferometer as biochemical sensor. Sensors and Actuators B, 18-19:183-187, 1994.

[2] A. Klotz, A. Brecht, and G. Gauglitz. Channel waveguide mode beat interferometer. Sensors and Actuators B, 38-39:310-315, 1997.

[3] C. Fattinger, H. Koller, D. Schlatter, and P. Wehrli. The difference interferometer: a highly sensitive optical probe for quantification of molecule surface concentration. Biosensors and Bioelectronics, 8:99-107, 1993.

[4] R.G. Heideman, R.P.H. Kooyman, and J. Greve. Polarimetric optical-fibre sensor for biochemical measurements. Sensors and Actuators B, 12:205-212, 1993.

[5] W. Lukosz, C. stamm, H.R. Moser, R. Ryf, and J. Dubendorfer. Difference interferometer with new phasemeasurement method as integrated-optical refractometer, humidity sensor and biosensor. Sensors and Actuators B, 38-39:316-323, 1997.

[6] C. Stamm and W. Lukosz. Integrated optical difference interferometer as immunosensor. Sensors and Actuators B, 31:203-207, 1996.

[7] R.G. Heideman and P.V. Lambeck. Remote opto-chemical sensing with extreme sensitivity: design, fabrication and performance of a pigtailed integrated optical phase modulated mach-zehnder interferometer system. Sensors and Actuators B, 61:100-127, 1999.

[8] K.Worhoff, A.Driessen, P.V.Lambeck, L.T.H.Hilderink, P.W.C.Linders, and T.J.A.Popma. Plasma enhanced chemical vapour deposition silicon oxynitride optimised for application in integrated optics. Sensors and Actuators $A, 74: 9-12,1999$.

[9] T.M.Koster and P.V.Lambeck. Fabrication tolerant passive polarisation converter realised in sion technology. In Proc. LEOS Benelux Chapter, 26 november, Gent, Belgium, 1998, pages 117-120, 1998.

[10] T.M.Koster and P.V.Lambeck. Fully integrated optical polarimeter. in preperation. 